\title{
Erfahrungen mit einem Laborprogramm zur Erfassung von Lupusantikoagulantien
}

\section{Report on a laboratory program for diagnosing lupus anticoagulants}

\author{
G. Lutze, K.-J. Hartung, H. Urbahn
}

Institut für Klinische Chemie des Universitätsklinikums der Otto-von-Guericke-Universität Magdeburg

\section{Zusammenfassung:}

Lupusantikoagulantien sind Autoantikörper verschiedener Immunglobulinklassen, die gegen Phospholipid-Protein-Komplexe gerichtet sind. Die Begriffe "Lupusantikoagulantien" (LA) und "Antiphospholipidantikörper" (APA) sind keine Synonyme, da es sich um unterschiedliche Antikörper handelt. Als LA sind nur diejenigen Antikörper zu bezeichnen, die phospholipidabhängige Gerinnungstests verlängern. Es werden Erfahrungen mit einem Laborprogramm mitgeteilt, das sich aus folgenden Abschnitten zusammensetzt:

- Screening für LA unter Verwendung von 2 APTT-Reagentien mit unterschiedlicher LA-Sensitivität

- Ausschluß von APTT-Verlängerungen, die nicht LA-bedingt sind

- Bestätigungsteste für $L A$

- Nachweis und Klassifizierung von APA

Dieses diagnostische Vorgehen trägt aufgrund der ausgeprägten Heterogenität der LA und APA nur zeitweiligen Charakter. Es ist unbekannt, mit welcher Vollständigkeit die benutzten Methoden die Erfassung von LA und APA gestatten. Weiterhin ist unbekannt, ob nur bestimmten LA- und APA-Untergruppen eine Pathogenität zuzuordnen ist oder ob LA und APA lediglich frühe Marker eines anderen thrombogenen Geschehens sind.

\section{Schlüsselwörter:}

Lupusantikoagulantien - Antiphospholipidantikörper - Labordiagnostik - aktivierte partielle Thromboplastinzeit

\section{Summary:}

Lupus anticoagulants are autoantibodies of different immunoglobulin classes directed against phospholipid-protein complexes. The termini "lupus anticoagulants" (LA) and "antiphospholipid antibodies" (APA) are not synonymous because LA and APA are different antibodies. Solely those antibodies should be named LA who causes prolongation of phospholipid-dependent tests of coagulation. Experiences with a laboratory program consisting of following sections are presented:

- screening for LA by using 2 APTT-reagents with different LA-sensitivity

- exclusion from APTT-prolongations not caused by $L A$

- confirmatory tests for $L A$

- detection and classification of APA

This approach is temporary due to the marked heterogeneity of LA and APA. It is unknown how completely these substances are determined by the used methods. Furthermore it is not yet clear wich subgroup of LA or/and APA has a pathogenic potentialor whether LA and APA occur merely as early markers of another thrombogenic happening.

\section{Keywords:}

Lupus anticoagulants-antiphospholipid antibodies-laboratory diagnostics-activated partial thromboplastin time 


\section{Einleitung}

1952 wurde erstmals von Conley und Hartmann ein $\mathrm{Zu}$ sammenhang $z$ wischen einer erhöhten Blutungsneigung und einer im Plasma kreisenden gerinnungshemmenden Substanz bei Patienten mit systemischem Lupus erythematodes beschrieben. 1972 schlugen Feinstein und Rapaport die Bezeichnung "Lupusantikoagulanz” für diese Substanz vor. Dieser auch heutzutage noch verwendete Terminus ist nach jetziger Kenntnis in zweifacher Hinsicht unglücklich. Einerseits ist die Mehrzahl der Patienten nicht an einem Lupus erythematodes erkrankt, andererseits besteht nur sehr selten eine erhöhte Blutungsneigung, wie es die Bezeichnung "Antikoagulanz" vermuten läßt, sondern es wird im Gegenteil ein gehäuftes Vorkommen von Thrombosen beobachtet.

Lupusantikoagulantien sind Autoantikörper verschiedener Immunglobulinklassen (lgG, IgM, IgA oder Mischungen), die gegen Phospholipid-Protein-Komplexe gerichtet sind (Antiphospholipidantikörper [APA]). Phospholipidbindende Proteine in solchen Komplexen sind $2 . B$.

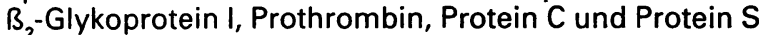
$(1-5)$.

Da Phospholipide für den lokalisierten Ablauf der Blutgerinnung unerläßlich sind, können Antikörper, deren Wirkung gegen Phospholipide gerichtet sind, auch den Gerinnungsablauf beeinflussen. Es handelt sich jedoch hierbei nicht um eine spezifische Reaktion mit definierten Gerinnungsfaktoren, wie dieses z.B. bei dem Faktor VIII-Antikörper der Fall ist, sondern um eine unspezifische gerinnungsverlangsamende Inhibitorwirkung auf phospholipidabhängige Gerinnungsabschnitte.

Die Begriffe „Lupusantikoagulanz" und „Antiphospholipidantikörper" sind nicht als Synonyme zu gebrauchen, da es sich um unterschiedliche Antikörper handelt. Als Lupusantikoagulantien (oder auch LupusantikoagulanzAktivität) sollte man Antikörper bezeichnen, die phospholipidabhängige Gerinnungstests pathologisch verlängern, nicht dagegen diejenigen APA, bei denen eine solche Lupusantikoagulanz-Aktivität fehlt. Zwar lassen sich bei der überwiegenden Mehrzahl der Patienten mit gerinnungsanalytisch nachgewiesener LupusantikoagulanzAktivität auch mit immunologischen Methoden APA nachweisen, bei einigen Patienten gelingt dies jedoch nicht. Andererseits können bei Patienten hohe APA-Konzentrationen bestehen, ohne daß die phospholipidabhängigen Gerinnungstests verändert werden $(6,7)$.

Aufgrund des eindeutigen Zusammenhangs zwischen Lupusantikoagulantien bzw. APA und Thrombosehäufigkeit sollte eine Thrombophilie- bzw. Thrombosediagnostik den Nachweis dieser Substanzen einschließen. Wertvoll ist dieser auch bei bestimmten geburtshilflichen und hämatologischen Fragestellungen, insbesondere bei gehäuften Aborten und intrauterinem Fruchttod sowie Thrombozytopenien (8-12).

Für die Diagnostik von Lupusantikoagulantien bzw. APA existieren eine Anzahl von Vorschlägen und Empfehlungen $(10,11,13-18)$.

In der folgenden Arbeit werden Ergebnisse mitgeteilt, die wir im Verlaufe mehrerer Jahre mit einem Laborpro- gramm erhalten konnten, das auf folgenden Abschnitten basiert:

1. Suchteste für Lupusantikoagulanz-Aktivität einschließlich Verifizierung einer unspezifischen Inhibitorwirkung

2. Bestätigungstests für Lupusantikoagulanz-Aktivität.

3. Bestimmungen und Klassifizierung von Antiphospholipidantikörpern (APA).

\section{Methodik}

Das Primärscreening auf Lupusantikoagulantien erfolgte im Rahmen unseres Basisprogramms zur Diagnostik von Gerinnungsstörungen. Es umfaßte folgende Untersuchungen: Thrombozytenzahl, Blutungszeit, Aktivierte Recalcifizierungszeit (ARZ), Thromboplastinzeit (TPZ, Quick-Test), Aktivierte Partielle Thromboplastinzeit (APTT), Thrombinzeit (TZ) und Fibrinogen.

- Thrombozytenzählung; Baker 9000+ (Serono Diagnostika GmbH, Freiburg i. Br.)

\section{- Blutungszeit; Methode nach Duke}

- Aktivierte Recalcifizierungszeit (ARZ) (19); Mischung von $0,5 \mathrm{ml}$ Citratblut mit $0,5 \mathrm{ml}$ Kaolin-CalciumchloridSuspension ( $1 \mathrm{~g} \mathrm{Kaolin} / 100 \mathrm{ml}$ 0,025 M CaCl ,-Lösung), manuelle Endpunktbestimmung

- Thromboplastinzeit (TPZ); Thromborel S (Behring AG, Marburg); zu Beginn der Untersuchungen wurden zusätzlich 2 weitere Thrombokinasen eingesetzt: Thrombokinase AWD (Arzneimittelwerk Dresden, jetzt nicht mehr im Handel), Hepato Quick (Boehringer Mannheim $\mathrm{GmbH}$ )

\section{- Aktivierte Partielle Thromboplastinzeit (APTT)}

- APTT 1 mit lupusantikoagulanz-insensitivem Reagenz; PTT-Reagenz (Boehringer Mannheim GmbH)

- APTT 2 mit lupusantikoagulanz-sensitivem Reagenz; Platelin Excel LS (Organon Teknika GmbH, Eppelheim)

- Thrombinzeit und Reptilasezeit; Thrombin-bzw. Reptilase-Reagenz (Boehringer Mannheim $\mathrm{GmbH}$ )

- Fibrinogen; Methode nach Clauss (Immuno GmbH, Heidelberg)

Einzelfaktorenbestimmungen wurden mit Reagentien der Immuno GmbH, Heidelberg, Behring AG, Marburg, und Boehringer Mannheim $\mathrm{GmbH}$ durchgeführt. Für den qualitativen Inhibitornachweis fand der Plasmatauschtest nach Deutsch Anwendung, als Bestätigungstest für Lupusantikoagulantien wurde der Lupus Anticoagulant Test der Immuno $\mathrm{GmbH}$, Heidelberg, eingesetzt.

Antiphośpholipidantikörper (APA)-Bestimmung wurden mittels ELISA vorgenommen: Asserachrom APA(Boehringer Mannheim GmbH); Asserachrom APA IgG/M (Boehringer Mannheim GmbH); QACA ELISA Kit (Cheshire Diagnostics Ltd.). 


\section{Ergebnisse}

\section{Suchteste für Lupusantikoagulanz-Aktivität}

Für diagnostische Belange besitzt die APTT besondere Bedeutung. Diese Reaktion kann aus verschiedenen Gründen pathologisch verlängert sein (Tabelle 1). Die von den verschiedenen Herstellern angebotenen Reagentien zur Durchführung der APTT-Reaktion differieren jedoch in ihrer Zusammensetzung. Dies betrifft sowohl die Art als auch die Konzentration der Phospholipid-und der Aktivatorkomponente. APTT-Verlängerungen aus den in Tabelle 1 dargestellten Gründen können aus diesem Grunde bei Untersuchung desselben Plasmas mit verschiedenen APTT-Reagentien sehr unterschiedliche Ergebnisse liefern. Lupusantikoagulantien werden daher je nach $\mathrm{Zu}$ sammensetzung des angewendeten APTT-Reagenzes mit unterschiedlicher Häufigkeit angezeigt. Für eine nähere, wenn auch unscharfe Charakterisierung der Reagentien befindet der Begriff der "Empfindlichkeit" Anwendung. Im Hinblick auf Lupusantikoagulantien bedeutet dies, daß "lupus-insensitive" von „lupus-sensitiven" Reagentien abgegrenzt werden können. Mit einem lupusinsensitiven APTT-Reagenz wird durch Lupusantikoagulantien in dem zu untersuchenden Plasma nur eine geringfügige oder keine Verlängerung der APTT festgestellt, mit einem lupus-sensitiven APTT-Reagenz dagegen eine ausgeprägte Verlängerung beobachtet.

Tab. 1: Ursachen von APTT-Verlängerungen.

1. Aktivitätsverminderungen von Faktoren des endogenen Gerinnungssystems

2. Heparin

3. Fibrin-Fibrinogen-Spaltprodukte

4. Inhibitoren

- inaktivierende Inhibitoren

- Lupusantikoagulantien

Diese Tatsache war der Ausgangspunkt für die Erprobung eines einfachen Lupusantikoagulanz-Screening, basierend auf APTT-Bestimmungen im Patientenplasma mit 2 APTT-Reagentien unterschiedlicher LupusantikoagulanzSensitivität. In Voruntersuchungen wurden APTT-Reagentien verschiedener Hersteller hinsichtlich ihrer "Lupusantikoagulanz-Sensitivität" untersucht. Prüfmaterial waren Plasmen von Patienten mit positiven Lupusantikoagulanz-Bestätigungstesten und-erhöhten APA-Konzentrationen. Insensitiv war das PTT-Reagenz der Boehringer

Tab. 2:APTT-Meßwerte von 3 Prüfmaterialien (Mittelwerte aus Doppelbestimmungen)

\begin{tabular}{|c|c|c|c|c|}
\hline & & & & \\
\hline & \multicolumn{2}{|c|}{ Manuelle Methode } & \multicolumn{2}{|c|}{ Kugelkoagulometer KC 40} \\
\hline & APTT 1 (s) & APTT 2(s) & APTT 1 (s) & APTT $2(s)$ \\
\hline &. & & . & \\
\hline Normalplasmapool & 32.6 & 33,4 & 31,5 & 32,6 \\
\hline & & . & & \\
\hline & & & & \\
\hline Preciclot I & 37,8 & 37,7 & 35,4 & 36,3 \\
\hline (Boehringer Mannheim) & & & & \\
\hline & & & $\therefore$ & \\
\hline Verify U normal & 37,5 & 39,1 . & 35,1 & 37,6 \\
\hline (Organon Teknika) & & & & $\cdots$ \\
\hline
\end{tabular}

Mannheim GmbH (APTT 1) - jedoch nicht das PTTa-Reagenz derselben Firma - als sehr sensitiv erwies sich Platelin Excel LS der Firma Organon Teknika GmbH, Eppelheim (APTT 2).

Bedingung für eine unmittelbare Vergleichbarkeit ist, daß bei gesunden Probanden nur geringe Meßwertunterschiede mit den beiden Reagentien auftreten. Die an 1 Normalplasmapool und 2 Kontrollmaterialien ermittelten Gerinnungszeiten sind in der Tabelle 2 dargestellt. Für das jeweilige Plasma differierten die Werte nur unwesentlich, wobei aber die mit APTT-Reagenz 2 erhaltenen Ergebnisse in den meisten Fällen geringfügig länger waren. Die Kontrollplasmen wurden bei jeder Untersuchung von Patientenplasmen mitgeführt, um bei Patienten auftretende Differenzen sicher beurteilen zu können.

In der Tabelle 3 sind Meßergebnisse der phospholipidabhängigen Globalteste und der Einzelfaktorenbestimmungen am Beispiel von 5 Patienten dargestellt. Der entscheidende diagnostische Hinweis auf ein Lupusantikoagulanz war die Differenz zwischen den Ergebnissen, die mit den beiden APTT-Reagentien erzielt wurden (s. auch Tabelle 7).

Ein unterstützender Befund bei der Diagnostik eines Lupusantikoagulanzes war das Verhalten der Aktivierten Recalcifizierungszeit (ARZ). Bei der überwiegenden Anzahl von Patienten wies sie eine Verlängerung auf, besonders wenn auch eine ausgeprägte Verlängerung der APTT 2 bestand. Die Thromboplastinzeit gab in den meisten Fällen keinen Hinweis auf das Vorliegen eines Lupusantikoagulanzes. Einige Thrombokinasen wiesen jedoch durch Erniedrigung des Thromboplastinzeitwertes auf einen Inhibitor hin, wie es in der Tabelle 3 am Beispiel der Humanhirnthrombokinase AWD gezeigt wird.

Bei Patienten mit Lupusantikoagulantien lagen die Einzelfaktorenaktivitäten trotz ausgeprägter APTT-Verlängerungen überwiegend in den Normalbereichen (Tabelle 3, Patienten 1 und 2).

Das Plasma mußte jedoch in mehreren Verdünnungen geprüft werden, um bisweilen vorhandene anscheinende Erniedrigungen durch Inhibitorsubstanzen zu erkennen. Bei einigen Patienten waren die Faktoren XI oder XII erniedrigt (Tabelle 3, Patienten 3-5).

Neben der ausreichenden Empfindlichkeit des APTTReagenzes auf Lupusantikoagulanz-Aktivität ist als zweiten Voraussetzung für den Einsatz der APTT-Reaktion zum LupusantikoagulanzScreening der Ausschluß der anderen Möglichkeiten, die eine APTT-Verlängerung hervorrufen, erforderlich.

Hierzu wurden folgende Untersuchungen durchgeführt:

- Thrombinzeit und Repti-
lasezeit

Sie dienten zur Erkennung der Anwesenheit von Heparin oder/und Fibrin-Fibrinogen- 
Tab. 3: Aktivierte Partielle Thromboplastinzeiten (APTT 1 und 2), Aktivierte Recalcifizierungszeit (ARZ), Thromboplastinzeiten (TPZ) und Einzelfaktorenaktivitäten bei 5 Patienten mit Lupusantikoagulantien

\begin{tabular}{|c|c|c|c|c|c|c|}
\hline & 1 & 2 & 3 & 4 & & \\
\hline APTा I (s) & $\$ 1,1$ & 47,6 & 48,1 & 46,2 & 46,8 & $\frac{\text { Normalberelche }}{<37,5^{\circ}}$ \\
\hline APTT 2(s) & 94,2 & 194,4 & 104,4 & 88,6 & 76,1 & $<40^{\circ}$ \\
\hline ARZ (s) & 127 & 190 & 107 & 116 & 146 & $<98$ \\
\hline & & & & & & \\
\hline TPZ (\%) & & & & & & $>70$ \\
\hline Thrombokinase AWD & 26 & 40 & 60 & 60 & 28 & \\
\hline Thromborel S & 75 & 70 & 80 & 70 & 33 & \\
\hline Hepato Qulck & 80 & 120 & 85 & 70 & 31 & \\
\hline Faktoren (\%) & & & & & & $>70$ \\
\hline II & 80 & 110 & 100 & 37 & 16 & \\
\hline$\underline{\mathbf{v}}$ & 105 & 115 & 140 & 120 & 80 & \\
\hline VII & 130 & 110 & 75 & 90 & 55 & \\
\hline$x$ & 70 & 125 & 80 & 110 & 48 & \\
\hline VIII & 120 & 115 & 150 & 100 & 105 & \\
\hline IX & 110 & 140 & 180 & 65 & 50 & \\
\hline $\mathbf{X I}$ & 100 & 260 & 46 & 46 & 38 & \\
\hline XII & 85 & 85 & 40 & 70 & 29 & \\
\hline "selbstermiltelte charg & ezlfisc & malbere & $(+2 s)$ & & & \\
\hline
\end{tabular}

Spaltprodukten (Tabelle 4). Die APTT-Reaktion konnte hierbei mit einem oder beiden Reagentien verlängert sein, die APTT 2 im Regelfall wesentlich ausgeprägter. Bei einer Hyperfibrinolyse (Fibrin-Fibrinogen-Spaltprodukte) waren Thrombinzeit und Reptilasezeit verlängert, bei Anwesenheit von Heparin nur die Thrombinzeit.

\section{- Aktivitätsbestimmungen der Einzelfaktoren VIII, IX, XI oder XII (Tabelle 5).}

Je nach Art des Faktors und Ausmaß der Aktivitätsverminderung traten mit beiden APTT-Reagentien Verlängerungen auf. Bemerkenswert war aber, daß die für Lupusantikoagulantien charakteristische ausgeprägte Differenz zwischen APTT 1 und APTT 2 bei Faktorenverminderungen nicht vorhanden war. Nicht selten war die Ursache der APTT-Verlängerung bekannt, so daß weitere Untersuchungen entfielen, z.B. bei bekannter Hämophilie oder Phenprocoumontherapie.

\section{- Inhibitornachweis}

Der Nachweis inaktivierender Inhibitoren erfolgte bei Faktorenerniedrigungen mittels Plasmatauschtest. Besondere Bedeutung hatte diese Untersuchung für die Erkennung von Faktor VIII-Inhibitoren.

Einige Besonderheiten sollen erwähnt werden:

\section{- Aktivitätsbestimmungen der Faktoren} II, V, VII und $X$

Die Bestimmung erfolgte bei den selten beobachteten zusätzlichen Erniedrigungen des Thromboplastinzeitwertes. In einigen Fällen - von uns jedoch nur bei
Vorliegen eines systemischen Lupus erythematodes gefunden - war der Faktor II erniedrigt (Tabelle 3, Patienten 4 und 5). Hierbei waren Prothrombinaktivität und-antigen gleichermaßen betroffen (20). Die niedrigsten von uns gemessenen Werte ergaben eine Prothrombinaktivität von $4 \%$ bei einem Prothrombinantigen von $5 \%$. Erniedrigungen weiterer Faktoren waren Ausnahmen (Tabelle 3, Patient 5) und dann durch eine echte faktorenbedingte Erniedrigung des Thromboplastinzeitwertes charakterisiert.

\section{- Thrombozytäre Störungen}

Diese waren oft jahrelang nachweisbar, wobei es sich um Thrombozytopenien 130 $95 \mathrm{Gpt} / \mathrm{l})$, Verlängerungen der Blutungszeit $(270-540 \mathrm{sec})$ und Störungen der ADP- und kollageninduzierten Aggregation handelte. Diese Patienten wiesen in vielen Fällen eine erhöhte Blutungsneigung auf.

Es soll noch einmal darauf hingewiesen werden, daß zur Beurteilung des Ergebnisses unseres Lupusantikoagulanz-Screenings die Zeitdifferenz zwischen APTT 1 und APTT 2 die entscheidende Rolle spielt. Nach unseren Erfahrungen sollte die Differenz zwischen den Ergebrissen mit den beiden zum Screening benutzten APTTReagentien mindestens 10-15 Sekunden betragen, wobei die APTT 2 länger als 40 Sekunden sein muß, wenn mit einem positiven Bestätigungstest gerechnet werden soll. Fiel das Screeningergebnis positiv aus, wurde das Plasma dem Bestätigungstest zugeführt.

\section{Bestätigungsteste für Lupusantikoagulanz-Aktivität}

Die in der Literatur beschriebenen Bestätigungsteste basieren auf der Wethselwirkung zwischen Lupusantikoagulantien und Phospholipiden. Sie variieren beträcht-

Tab. 4: Gerinnungsanalytische Befunde von 6Patienten mit Heparin- und Streptokinasetherapie (Einzelfaktoren im Normbereich, nicht aufgeführt)

\begin{tabular}{|c|c|c|c|c|l|}
\hline & & & & \\
\hline & & & & & \\
\hline Patient & APTT 1 & APTT 2 & Thrombinzeit & Reptilase- & Beurteilung \\
\hline & & & & zeit & \\
\hline & $(\mathrm{s})$ & $(\mathrm{s})$ & $(\mathrm{s})$ & $(\mathrm{s})$ & \\
\hline & & & & & \\
\hline 1 & 37,4 & 78,3 & 106,6 & 19,8 & Heparin \\
\hline 2 & 52,4 & 119,9 & 84,2 & 19,6 & Heparin \\
\hline 3 & 165,6 & $>300$ & 110,1 & 20,4 & Heparin \\
\hline & & & & & \\
\hline 4 & 29,4 & 37,1 & 35,6 & 30,8 & Hyperfibrinolyse \\
\hline 5 & 39,4 & 42,6 & 42,4 & 60,4 & Hyperfibrinolyse \\
\hline 6 & 44,5 & 68,4 & 31,6 & 28,1 & Hyperfibrinolyse \\
\hline
\end{tabular}


lich in ihrer-Durchführung, wobei zwei Testprinzipien existieren (Tabelle 6). BeiAnwendung verminderter Phospholipidkonzentrationen wird der Inhibitoreffekt akzentuiert, hohe Phospholipidkonzentrationen neutralisieren den Inhibitoreffekt.

Wir setzten routinemäßig den Lupus Anticoagulant Test der Immuno $\mathrm{GmbH}$, Heidelberg, ein. Hierbei wurden zwei APTT-Bestimmungen mit unterschiedlichen Phospholipidkonzentrationen (test system with decreased phospholipid) in einem Normalplasma, dem Patientenplasma und einer $1+1$ - Mischung aus beiden durchgeführt. Die graphische Darstellung und der LCA-Index ( $L C A=$ Lupus Circulating Anticoagulant Activity) erlaubten eine Beurteilung. Der LCA-Index errechnete sich aus den mit der Phospholipidkonzentration 2 (PL 2) erhaltenen Meßwerten der 3 Untersuchungsproben:

$$
\text { LCA-Index }=\frac{t 50 \%(s)-t 0 \%(s)}{t 100 \%(s)} \times 100
$$
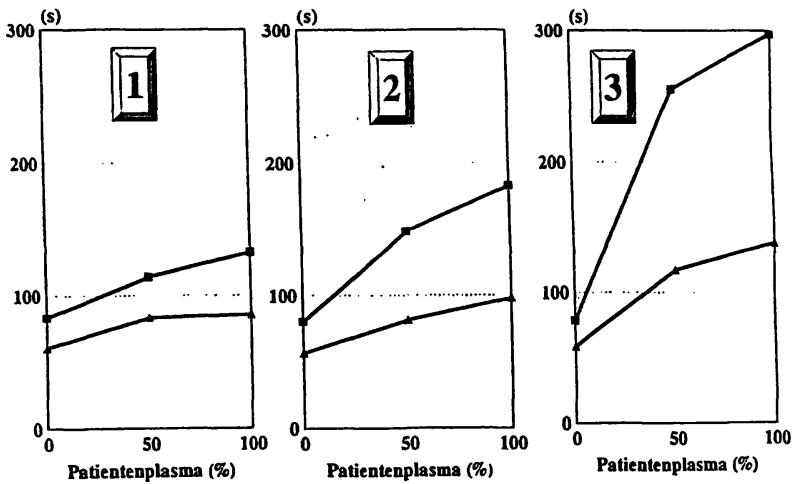

Abb. 1: Positive Lupus Anticoagulant Tests bei 3 Patienten ( $\triangle$ PL 1; a PL 2 [1:31])

LCA-Indices: Patient $1=23,5 ;$ Patient $2=37,2 ;$ Patient $3=59,6$

Tab. 5: Gerinnungsanalytische Befunde von 4 Patienten mit Hämophilie (TZ, RZ und TPZ im Normbereich, nicht aufgeführt)

\begin{tabular}{|l|r|r|r|r|r|r|r|l|}
\hline & & & & & & & \\
\hline Patient & APTT 1 & APTT 2 & VIII & IX & XI & XII & & Beurteilung \\
\hline & (s) & (s) & $(\%)$ & $(\%)$ & $(\%)$ & $(\%)$ & & \\
\hline & & & & & & & & \\
\hline 1 & 89,4 & 95,9 & $<1$ & 120 & 115 & 95 & & Hämophilie A (schwer) \\
\hline 2 & 53,6 & 57,1 & 8 & 95 & 90 & 90 & & Hämophilie A (leicht) \\
\hline & & & & & & & & \\
\hline 3 & 133,6 & 140,1 & 95 & $<1$ & 120 & 85 & & Hämophilie B (schwer) \\
\hline 4 & 53,4 & 57,4 & 100 & 9 & 80 & 115 & & Hämophilie B (leicht) \\
\hline
\end{tabular}

Tab. 6: Bestätigungsteste für Lupusantikoagulanz-Aktivität (13)

Bei Anwesenheit von Substanzen mit Lupusantikoagulanz-Aktivität war der Kurvenverlauf konvex und der LCA-Index erreichte Werte über 15,0. Der LCA-Index hatte insbesondere für Verlaufskontrollen Bedeutung:

In der Abbildung 1 sind Kurvenverläufe von 3 Patienten dargestellt, die eine konvexe Form aufweisen und bei denen der LCA-Index oberhalb von 15,0 lag. Dieses Ergebnis bedeutete das Vorhandensein von Lupusantikoagulanz-Aktivität in den untersuchten Plasmen.
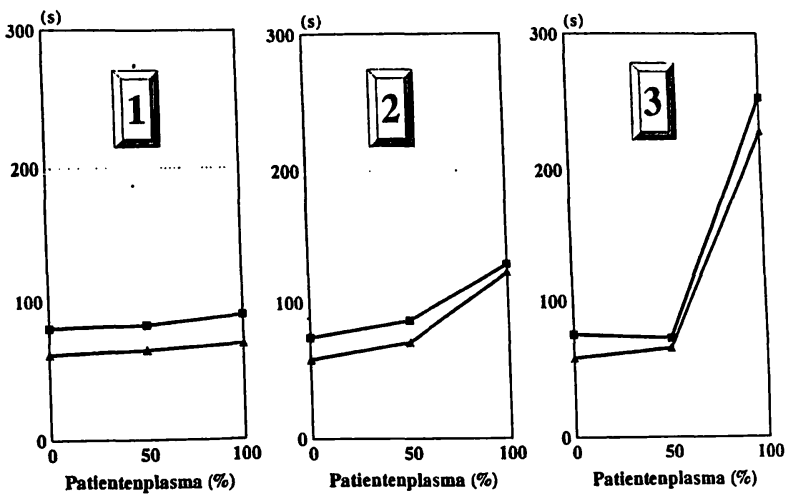

Abb. 2: Negative Lupus Anticoagulant Tests bei 3 Patienten (A PL 1; 口 $P L$ 2 [1:31])

LCA-Indices: Patient $1=2,2 ;$ Patient $2=9,4 ;$ Patient $3=<0,09$

In der Abbildung 2 sind Ergebnisse von 3 Patienten dargestellt, deren Kurvenverläufe eine LupusantikoagulanzAktivität ausschlossen. Die Kurvenform war konkav, und die LCA-Indices lagen unter 15,0. Solche negativen Ergebnisse bei verlängerter APTT traten bei Faktorenmangel auf, aber auch bei inaktivierenden Inhibitoren (Hemmkörperhämophilie A bzw. B), wobei erwähnt werden muß, daß wir bei zwei Patientinnen mit spontanem Faktor VIII-Inhibitor und 1 Patienten mit Hemmkörperhämophilie A zeitweise zusätzlich auch eine Lupusantikoagulanz-Aktivität nachweisen konnten.

Bei einigen Plasmen, war der Screeningtest falsch positiv (APTT-Differenz > 10 Sekunden bei negativem Bestätigungstest). Die Ursache für nicht zu bestätigende positive Screeningergebnisse konnten wir bisher nicht immer finden. Eine wichtige Rolle scheinen geringe Aktivitätsverminderungen der Faktoren VIII, IX, XI, besonders aber des Faktors XII zu spielen. Die mehrfach ermittelten Faktor XII-Erniedrigungen auf $30-50 \%$ der Norm können infolge einer höheren Empfindlichkeit des APTT 2Reagenzes auf eine Faktor XII-Verminderung das positive Screeningergebnis erklären.
Test Systems with Decreased Phospholipid

Tissue Thromboplastin Inhibition (TTI)

Dilute Russell Viper Venom Time (dRVVT)

Kaolin Clotting Time (KCT)

Dilute Phospholipid APTT

Test Systems with Increased Phospholipid

Platelet Neutralization Procedure (PNP)

High Phospholipid APTT

Rabbit Brain Neutralization

Phosphatidyl Serine Liposome APTT

Hexagonal Phospholipid Neutralization 


\section{Antiphospholipidantikörper-Bestimmungen}

Der 3. Abschnitt der Labordiagnostik beinhaltete den Nachweis von Antiphospholipidantikörpern bzw. deren quantitative Bestimmung und Klassifizierung. Der semiquantitative Antikörpernachweis ohne Klassifizierung erfolgte mit Asserachrom APA.

Es handelte sich um einen ELISA, bei dem die Antiphospholipidantikörper an ein Phospholipidgemisch aus Cardiolipin, Phosphatidylserin und Phosphatidsäure gebunden werden. Die semiquantitative Auswertung erfolgte sowohl mit negativen, positiven als auch cut-offKontrollplasmen.

Die quantitative Bestimmung der Antiphospholipidantikörper und ihre Klassifizierung erfolgte mit Asserachrom APA IgG, M (ELISA mit Gemisch aus Cardiolipin, Phosphatidylserin und Phosphatidsäure) und OACA ELISA (ELISA mit Cardiolipin).

In Tabelle 7 sind Meßwerte von 12 Patienten zusammengestellt. Neben dem APTT-Screening wurden als Ausdruck der Lupusantikoagulanz-Aktivität die LCA-Indices

Mit den verwendeten Testkits Asserachrom APA bzw. QACAELISA konnten prinzipiell vergleichbare Aussagen getroffen werden, wenn auch die Zahlenwerte teilweise stark differierten.

\section{Diskussion}

Da die Kenntnis über die Anwesenheit von Lupusantikoagulantien bzw. Antiphospholipidantikörpern bei einer Anzahl von Patienten eine wesentliche Bedeutung für die Prognose, Prophylaxe und Therapie der möglichen Komplikationen besitzt, muß eine häufigere und qualitativ bessere Durchführung der Diagnostik angestrebt werden. Obwohl eine Vielzahl von Labortesten beschrieben wurde, kann bisher nicht eindeutig entschieden werden, welche Methode bzw. Methodenkombination die geeigneteste für die Diagnostik ist, da es sich bei den Lupusantikoagulantien und APA um sehr heterogene Substanzgruppen handelt. Es scheint jedoch gesichert, daß es sich um zwei distinktive Antikörpergruppen handelt, wahrscheinlich auch mit eigenständiger Pathogenität. Daher sind Lupusantikoagulantien und APA gesondert und unabhängig voneinander zu diagnostizieren:

Tab. 7:APTT-Screening, Lupusantikoagulanz-Aktivität (LCA-Indices) und AntiphospholipidantikörperKonzentration bei 12 Patienten

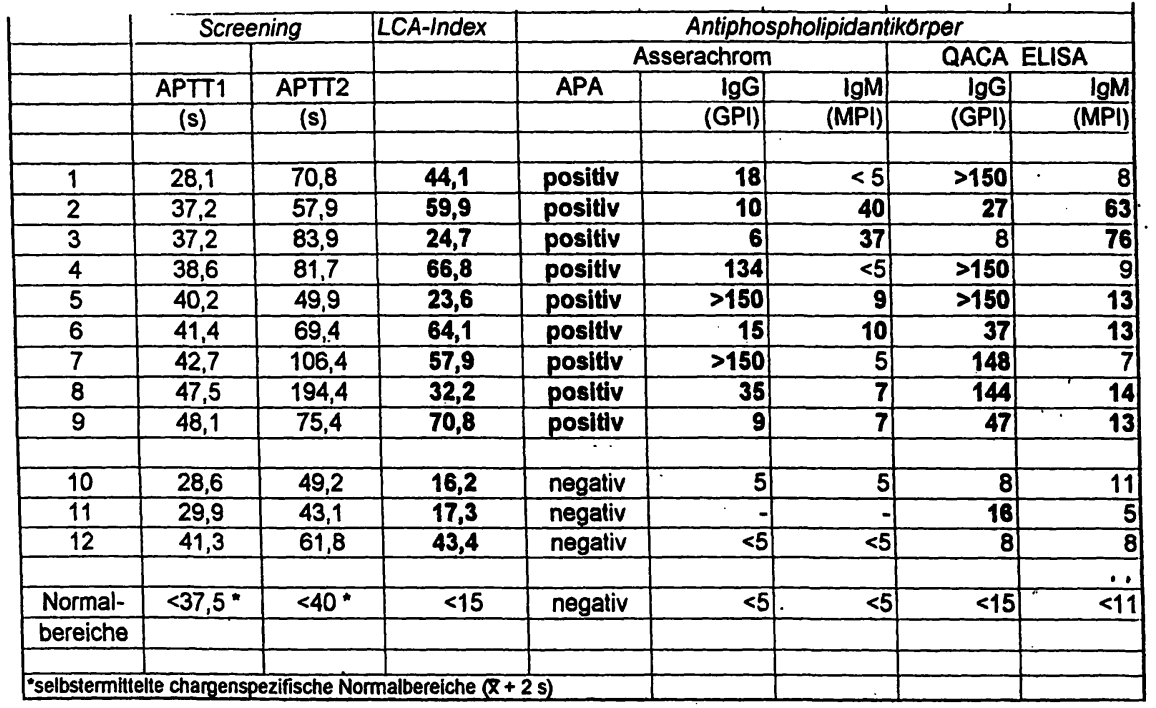

- Lupusantikoagulantien durch Screening- und Bestätigungstests nach Ausschluß interferierender Möglichkeiten,

- Antiphospholipidantikörper mittels Immunoassays (2123).

Hiermit werden 3 Patientengruppen erfaßt: Patienten mit Lupusantikoagulanz-Aktivität kombiniert mit APA (überwiegend), Patienten mit isolierter Lupusantikoagulanz-Aktivität oder isolierten höheren APAKonzentrationen (seltener).

Ein brauchbares Screening auf Lupusantikoagulantien muß im Rahmen eines gerinnungsanalytischen Basisprogramms schnell und unkompliziert durchführbar sein. Unser Screening auf Lupusantikoagulanz-Aktivitätbasiert

aufgeführt, die sämtlich im pathologischen Bereich lagen.

Bei den Patienten 1-9 waren die Erhöhungen des LCAIndex mit APA-Erhöhungen gekoppelt, nicht jedoch bei den Patienten 10-12. Während die Patienten 10 und 11 als Grenzwerte aufgefaßt werden können, zeigte Patient 12 bei eindeutig positivem Lupusantikoagulanztest keine APA-Erhöhung, auch nicht bei mehreren Kontrolluntersuchungen. Die APA-Erhöhungen konnten sowohl die IgG-als auch die IgM-Klasse betreffen. Besonders ausgeprägte Erhöhungen traten bei der lgG-Klasse auf. Eine Korrelation zwischen Lupusantikoagulanz-Aktivität und APA-Konzentration bestand jedoch nicht. auf der APTT-Reaktion. Die Untersuchungen zeigten, daß mit dieser Reaktion ein Lupusantikoagulanz-Screening erfolgversprechend ist, wenn 2 Reagentien mit möglichst stark differierender Lupusantikoagulanz-Sensitivität verwendet werden. Die höchste Empfindlichkeit scheint das speziell für die Lupusantikoagulanzdiagnostik entwickelte APTT-Reagenz PTT-LA der Firma Diagnostica Stago, Asnieres-sur-Seine, France, aufzuweisen (24).

Nach Ausschluß anderer Ursachen für APTT-Verlängerungen (Tabelle 1) müssen Bestätigungsteste für Lupusantikoagulanz-Aktivitäten mit verminderten oder erhöhten Phosphoipidkonzentrationen angeschlossen werden. Aufgrund der Heterogenität der Lupusantikoa- 
gulantien ist die Anwendung von einemBestätigungstest nicht ausreichend. Es ist die gleichzeitige Anwendung beider Testprinzipien zu erwägen, d.h. in Ergänzung zu unserer bisherigen Verfahrensweise (decreased phospholipid test system) wäre ein high phospholipid test system, z.B. Staclot LA (Stago), anzuschließen (Tab. $6 ; 13,25)$.

APA-Bestimmungen sollten nicht als letzte Stufe der Diagnostik bei nachgewiesener Lupusantikoagulanz-Aktivität betrachtet werden, sondern zum obligatorischen Bestandteil eines aus klinischer Indikation durchgeführten Untersuchungsprogramms („Thromboseprogramm") gehören. Eine Erfassung der APA ohne Lupusantikoagulanz-Aktivität, die sonst nicht möglich ist, erfolgt hiermit und trägt eventuell zur Klärung bestimmter thromboembolischer Ereignisse oder Risiken bei.

Der gleichzeitige Einsatz von Testkits, die sich in wesentlichen Komponenten, z.B. im Phospholipid, unterscheiden, sollte erwogen werden, auch wenn unsere Ergebnisse mit prinzipiell übereinstimmenden Aussagen trotz unterschiedlicher Phospholipidzusammensetzung der Testkits nicht unbedingt für die Notwendigkeit eines solchen Vorgehens sprechen (Tabelle 7).

Es muß darauf hingewiesen werden, daß eine befriedigende Lösung der beschriebenen Problematik gegenwärtig noch nicht möglich ist. Das dargestellte diagno- stische Vorgehen trägt daher nur zeitweiligen Charakter. Eine effiziente Diagnostik hat eine Klärung folgender Fragen zur Voraussetzung:

1. Aufgrund der Heterogenität der Lupusantikoagulantien und APA ist es nicht bekannt, mit welcher Vollständigkeit bisher die Erfassung erfolgt. Dieses betrifft sowohl Screening- und Bestätigungsteste für Lupusantikoagulanz-Aktivität als auch die APA-Bestimmungen. Es wird allgemein akzeptiert, daß eine Kombination mehrerer Untersuchungen bei jedem diagnostischen Schritt empfehlenswert ist, die beste Kombination ist jedoch unbekannt (26). Die zahlreichen Empfehlungen von Methoden und Methodenkombinationen mit der höchsten diagnostischen Erfolgsquote sind mit Vorsicht zu beurteilen, da neben den Methoden auch die Art der Erkrankungen, die Auswahlprinzipien der Patienten sowie andere Einflüsse eine wesentliche Rolle spielen können.

2. Es ist bisher nicht bekannt, welchen APA bzw. APAProtein-Komplexen eine Pathogenität zuzuordnen ist. Eine Standardisierung und Vervollkommnung der $\mathrm{Me}$ thodik sollte nur die wirklich relevanten Antikörper einbeziehen $(23,26)$.

3. Es ist letztendlich nicht bekannt, ob die APA selbst bzw. ihre Komplexe Pathogenität besitzen oder ob sie nur frühe Marker eines anderen thrombogenen Geschehens sind, z.B. der Schädigung bestimmter zellulärer Strukturen. 


\section{Originalie}

Literatur:

1. McNeil, H.P.; Simpson, R.J.; Chesterman, C.N.; Krilis, S.A. (1990): Antiphospholipid antibodies are directed against a complex antigen that includes a lipid-binding inhibitor of coagulation: B2-Glycoprotein I (apolipoprotein H). Proc. Natl. Acad. Sci. USA 87, 4120.4124.

2. Rauch, J.; Janoff, A.S. (1990): Phospholipid in the hexagonal II phase is immunogenic. Proc. Natl. Acad. Sci. USA 87, 4112-4114.

3. Bevers, E.M.; Galli, M.;Barbui, T.; Comfurius, P.;Zwaal,R.F.A. (1991): Lupus anticoagulant IgG's (LA) are not directed to phospholipids only, but to a complex of lipid-bound human prothrombin. Thromb. Haemostas. $66(6)$ 629-632.

4. Galli, M.; Comfurius, P.; Barbui, T.; Zwaal, R.F.A.; Bevers, E.M. (1992): Anticoagulant activity of $B 2-$ Glycoprotein $I$ is potentiated by a distinct subgroup of anticoardiolipin antibodies. Thromb. Haemostas. 68, 297-300.

5. Oosting, J.D.; Derksen, R.H.W.M.; Bobbink, I.W.G.; Hackeng, T.M.; Bouma, B.N.; de Groot, P.G.; (1993): Antiphospholipid antibodies directed against combination of phospholipids with prothrombin, protein C or protein S - an explanation for their pathogenic mechanism? Blood 81 (10), 2618-2625.

6. Ames, P.R.J.; Brancaccio, V.; lannaccone, L.; Piemontino, U.; Maresca, G. Di Minno, G. (1993): Hemostatic variables, antiphospholipid antibodies and thrombosis. A survey of 133 patients. Thromb. Haemostas. 69 (6), 1220 (Abstr. 2412).

7. Biasiolo, A.; Banzato, A.; Garelli, E.; Zasso, A.; Pengo, V. (1993): Prevalence of antiphospholipid antibodies in patients with venous thromboembolic disease. Thromb. Haemostas. 69 (6), 1226 (Abstr. 2433).

8. Branch, D.W.: Scott, J.R.; Kochenour, N.K.; Hershgold, E. (1985): Obstetric complications associated with the lupus anticoagulant. NewEngl.J. Med.313 (21), 1322-1326

9. Triplett, D.A. (1988): Clinical significance of antiphospholipid antibodies. Thromb. Haemostas. 10 (1), 1-10.

10. Kolde, H.-J. (1989): Lupus-Antikoagulanzien - ein unterschätzter Risikofaktor für die Entwicklung von thrombotischen Erkrankungen? Extr. diagn. 3 (6), 286-289.

11. Reimann, U. (1991): Paradoxe Thrombosen. Diagnostica Dialog 6/91.

12. Bick, R.L. (1993): Lupus anticoagulants and anticardiolipin antibodies. Biomed. Progr. 6, 35-39.

13. Triplett, D.A. (1989): Screening for the lupus anticoagulant. Res. Clin. Lab. 19, 379-389.

14. Exner, T.; Triplett, D.A.; Taberner, D.; Machin, S.J. (1991): Guidelines for testing and revised criteria for lupus anticoagulants, Thromb. Haemostas. 65 (3), 320-322.

15. Saxena, R.; Saraya, A.K.; Kotte, V.K.; Singh, Y.N.; Prasad, L.; Malviya, A.N. (1991): Evaluation of four coagulation tests to detect plasma lupus anticoagulants. Am. J. Clin. Pathol. 96 (6), 755-758.

16. Triplett, D.A. (1992): Coagulation assays for the lupus anticoagulant: review and critique of current methodology. Stroke 23 (Suppl. I) 11-14.
17. Triplett, D.A. (1993): Laboratory investigation of phospholipid-binding antibodies and lupus anticoagulants. International Stago symposium on phospholipid-binding antibodies and thrombosis, Paris.

18. Halbmayer, W.-M.; Haushofer, A.; Schratzberger, W.; Petera, P.; Duschet, P.: Fischer, M. (1993): Nachweis von Lupusantikoagulanzien im Routinegerinnungslabor am Beispiel von 36 Patienten mit systemischem Lupus erythematodes. Med. Klinik 88, 417-422.

19. Lutze, G.; Urbahn, H. (1981): Bestimmung der Aktivierten Rekalzifizierungszeit (ARZ) im Zitratblut. Z. med. Labor.-Diagn. 22, 37-42.

20. Baudo, F.; Redaelli, R.; Pezzetti, L.; Caimi, T.M.; Busnach, G.; Perrino, L.; de Cataldo, F. (1990): Prothrombin-antibody coexistent with lupus anticoagulant (LA): clinical study and immunochemical characterization. Thromb. Res. 57 (2), 279-287.

21. De Moerloose, P.; Reber, G.; Vogel, J.-J. (1990): Anticardiolipin antibody determination: comparison of three ELISA assays. Clin. Exp. Rheumatol. 8. 575-577.

22. Amiral, J.; Minard,F.; Chambrette, B. (1991): Development of standardized immunoassays for identification, characterization and quantitation of antiphospholipid antibodies (APA). Biol. Clin. Hematol. 13, 81-88.

23. De Moerloos, P. (1993): Rationale of the standardization of the detection of phospholipid-binding antibodies by solid-phase assays. International Stago symposium on phospholipid-binding antibodies and thrombosis, Paris.

24. Cauchie, P.; Verheilwheghen, V.; Brohee, D.; Lefévre, A. (1993): High sensitivity of a new APTT reagent in lupus anticoagulant detection. Thromb. Haemostas. 69 (6), 1035 (Abstr. 1752).

25. Triplett, D.A.;Hannah, D.;Barna, L. (1991): Hexagonal (II) phase phospholipid neutralization of lupus anticoagulants: a specific and sensitive confirmatory procedure. Thromb. Haemostas 65 (6), 1255 (Abstr. 2021).

26. Sie, P. (1993): Rationale of the standardization of the detection of lupus anticoagulants. International Stago symposium on phospholipid-binding antibodies and thrombosis, Paris.

\section{Anschrift des Verfassers:}

Prof. Dr. G. Lutze

Otto-von-Guericke-Universität

Medizinische Fakultät

Institut für Klinische Chemie

Leipziger Straße 44

39120 Magdeburg 